\title{
Crisis agrícola y pluriactividad: mujeres trabajando en la industria textil y de confección en Tepetitla de Lardizábal, Tlaxcala
}

Agricultural crisis and pluriactivity: woman working in textile and clothing industry in Tepetitla de Lardizábal, Tlaxcala.

\author{
Janett Vallejo Román*
}

\section{Resumen}

El objetivo general de este trabajo es presentar algunos hallazgos en torno al análisis del mercado de trabajo rural enmarcado en la pluriactividad, partiendo del análisis de las transformaciones producto de la globalización neoliberal y la crisis agrícola en México. En un nivel más particular, se trata de poner en la mesa de discusión la complejidad de los procesos de negociación (cooperación y conflicto) que se derivan del "deseo" o decisión de las mujeres de trabajar en actividades extradomésticas y fuera del hogar. Se recupera el trabajo de las mujeres que trabajan en talleres de confección de mezclilla en el municipio de Tepetitla de Lardizábal, Tlaxcala, México

Palabras clave: Globalización, ruralidad, pluriactividad, trabajo, género.

\begin{abstract}
The main goal of this paper is to discuss some findings about rural labor work framed in pluriactivity, given main changes produced by neoliberal globalization and rural crisis in Mexico. Specifically, It is discussed the complex negotiation processes (cooperation and conflict) because of the women's "wish" or decision to work out of home or in nondomestic activities. The women's work in denim workshops at Tepetitla de Lardizábal, Tlaxcala, Mexico is highlighted.
\end{abstract}

Key words: Globalization, rural life, pluriactivity, work, gender.

Fecha de recepción: 01 de septiembre 2017

Fecha de aprobación: 04 de abril 2017

\footnotetext{
* Doctora en Geografía y posdoctorante en Antropología por la Universidad Nacional Autónoma de México, México. Investigación realizada gracias al apoyo otorgado por el Programa de Becas Posdoctorales de la UNAM y el proyecto "Ruralidades, sujetos sociales y respuestas comunitarias" (PAPIIT IN 300115), Responsable Hernán Salas.
} 


\section{Introducción}

En la actualidad, los hogares rurales en México dependen cada vez más de ingresos provenientes de actividades distintas a las agrícolas, además requieren de la inserción de sus distintos miembros al mercado de trabajo no agrícola para la obtención de los recursos mínimos para su sobrevivencia. Particularmente, se observa la inserción de las mujeres al trabajo fuera de casa, hecho que ha generado importantes cambios en la organización familiar.

Durante largo tiempo el trabajo de la mujer fue reconocido como una actividad adicional al realizado por el jefe de familia, ya que en términos económicos no representaba el principal ingreso del hogar. Hoy en día, el ingreso proveniente del trabajo de las mujeres no puede ser considerado como "complementario", porque en un número creciente de hogares el sustento principal proviene de las remuneraciones económicas de una o varias mujeres, incluso en aquellos con jefatura masculina.

La incorporación de las mujeres al mercado de trabajo no sólo ha puesto en la discusión la invisibilidad con la que por décadas se trató al trabajo femenino; también centra el debate en las relaciones de poder dentro del hogar, así como en las formas de negociación, las cuales, en su mayoría, atraviesan por el conflicto y tensiones, y que día con día se redefinen o afianzan en las relaciones familiares.

El objetivo general de este trabajo es presentar algunos hallazgos en torno a las trasformaciones del mercado de trabajo rural partiendo del análisis de los cambios producto de la globalización neoliberal y la crisis agrícola en México; en un nivel más particular, se trata de discutir la inserción de las mujeres a dicho mercado y los cambios familiares que esto ha implicado. Se recupera el caso de un municipio rural en el Centro de México: Tepetitla de Lardizábal, Tlaxcala. Este municipio se caracteriza por su alta especialización en la producción de pantalones de mezclilla a través de talleres familiares de diversos tipos y tamaños que operan en su gran mayoría de manera informal y clandestina.

La metodología utilizada es de carácter mixto (Hernández et al., 2003) dando preferencia al trabajo etnográfico. Se contrastó la información cuantitativa, tomada de documentos e informes oficiales gubernamentales y de la aplicación de un cuestionario, con la información cualitativa recogida en el trabajo de campo llevado a cabo durante el 2014, 2015 y 2016 por medio de observación directa y entrevistas semiestructuradas. Los talleres establecidos en el caso de estudio, como se mencionó son informales y clandestinos, por lo que en principio dificultó la obtención de información, sin embargo, las estancias prolongadas de campo permitieron recabar información de primera mano con informantes claves como dueños de talleres, trabajadores agrícolas y en los talleres, así como comerciantes del pantalón. 
Este trabajo se presenta de la siguiente manera: primero se aborda en términos generales los cambios económicos globales y sus repercusiones locales; en un segundo apartado se presenta el tema de la crisis agrícola en México y la pluriactividad como una estrategia de sobrevivencia en los espacios rurales; en el tercer y cuarto apartado se recupera el caso de Tepetitla de Lardizal, enfatizando la inserción de las mujeres en el trabajo de los talleres familiares de confección. El trabajo concluye con algunas reflexiones generales.

\section{Cambios globales repercusiones locales}

Las transformaciones en el ámbito local no pueden ser explicadas únicamente por los cambios endógenos de las comunidades, se requiere de una mirada más amplia que cruce diversas escalas. Como señala Echánove y Riedemann (2005: 30) lo ocurrido a escala local se transforma en la medida en que forma parte de esferas y procesos globales, mientras que la dimensión global se constituye fundamentalmente en relación con condiciones locales específicas y con las distintas estrategias de los actores locales. Así, el análisis de la relación global-local se muestra imprescindible en los estudios económicos y sociales actuales

En el plano económico, asistimos a una fase de cambios acelerados y profundos que afectan el funcionamiento de las naciones teniendo implicaciones espaciales concretas, tales como: la progresiva apertura y ampliación de los mercados; la redistribución del trabajo; y la localización del capital en territorios con mayor capacidad para atraer o generar iniciativas empresariales, entre otras.

En esta investigación se parte del análisis del fenómeno de la globalización neoliberal, entendida como un proceso de reestructuración económica, política y cultural mundial. Particularmente en el ámbito económico, el proceso de globalización ha gestado cambios importantes en los procesos mundiales de trabajo y producción basados en la nueva división internacional de trabajo (NDIT) sostenida por la barata y abundante fuerza de trabajo en los países en desarrollo, la fragmentación de los procesos productivos y el avance tecnológico en materia de transportes e informática así como por la fragmentación de la producción.

En este contexto, el territorio se ha revalorizado principalmente en el ámbito local y regional. La conformación de un nuevo tejido productivo, apoyado por redes de producción nacional e internacional y transacciones financieras, se traduce en la creación de nuevos mercados de trabajo articulados y jerarquizados. Es aquí en donde los espacios regionales y locales funcionan como ejes articuladores de la economía global. No obstante, es importante señalar que este proceso de articulación económica, tiene efectos diferenciados, hay regiones y localidades "ganadoras" y "perdedoras"; esto tiene como consecuencia el incremento de disparidades sociales y económicas. 
Como menciona García (2001:58) un rasgo principal de la reestructuración económica ha sido la búsqueda de mano de obra barata y flexible que permita lograr de manera rápida la competitividad internacional. Esto ha significado cambios en la configuración de mercados de trabajo y de la fuerza de trabajo en el medio rural y urbano por igual. Sin embargo, se puede observar que en los espacios rurales hay una tendencia importante hacia la creación de nuevos mercados de trabajo.

Por medio de la fragmentación y difusión de los procesos de producción, asociada a los paradigmas de flexibilidad, se han favorecido las posibilidades de dispersión territorial de las empresas. Por ejemplo, en el caso de las maquiladoras han sido impulsadas por la reestructuración productiva e intensificadas por las empresas industriales transnacionales, y en algunos casos nacionales, para enfrentar la crisis de largo plazo del capitalismo. Así, éstas aparecen como un eslabón de la cadena productiva industrial y como una estrategia económica de la empresa global (Morales 2000:10.) en donde los espacios rurales han funcionado como anclajes territoriales.

En la zona de estudio, particularmente en el municipio de Tepetitla de Lardizábal, la fragmentación de la producción promovida por las grandes empresas ha sido adoptada, adaptada y reproducida por los medianos y pequeños talleres instalados de manera formal o informal para la producción local y regional. Es decir, el modelo de producción instrumentado por las grandes trasnacionales y nacionales de la confección ha sido recuperado por los pobladores como una medida para hacer frente a la crisis agrícola y de la industria textil tradicional tlaxcalteca. Encontrando en esta nueva forma de operar de la industria textil y de la confección una alternativa de sobrevivencia.

La instalación de maquiladoras en sus distintas modalidades y escalas, obedece, entre otras cosas, al impulso gubernamental de actividades industriales en los espacios rurales y periurbanos. El presupuesto es que en estos espacios existen ventajas competitivas como la disposición de suelo, mano de obra abundante, adecuada localización geográfica, etc. que son idóneas para la ejecución de políticas públicas favorables para la "industrialización", con lo cual se supone, se detonará el "desarrollo" económico en dichos espacios. En el caso de estudio, el municipio de Tepetitla, que hasta los años ochenta podía considerarse como un espacio de producción agrícola, ha reducido esta actividad considerablemente y se ha reconvertido casi en su totalidad a la actividad industrial en pequeña escala. A pesar de la presencia industrial, aún se observa producción agrícola para el autoconsumo, pero ésta es cada vez menor, son las generaciones mayores quienes aún conservan el interés por sembrar. Los jóvenes e incluso personas cuya edad promedio oscila entre los 40 y 50 ya no encuentran viable continuar con la siembra. Así lo menciona un entrevistado, "la siembra ya no es negocio, ni te deja para vivir. Inviertes mucho y ganas poco o nada. Eso se lo dejamos a los viejos para que se entretenga. Nosotros, aquí en el taller" (Enrique, Tepetitla, 2014) 
En esta localidad no existe la instrumentación de políticas públicas explícitas o programas de desarrollo económico que estimulen el desarrollo de los talleres de confección y lavanderías, sin embargo, es un hecho que existe una postura de tolerancia que ha favorecido el establecimiento y consolidación de estas actividades. Los talleres operan sin ningún tipo de permiso o reglamento oficial. Las autoridades gubernamentales en sus distintos niveles dejan operar los cientos de talleres y lavanderías, y es que pesé a las precarias condiciones laborales en los talleres y los problemas ambientales que estos han generado, la oferta de empleos es considerable. En este sentido, los talleres familiares han funcionado como un mecanismo de amortiguamiento ante la crisis agrícola y la falta de oportunidades laborales y escolares, además de ser una estrategia de retención e incluso atracción de población.

\section{La crisis agrícola y la pluriactividad como alternativa de sobrevivencia}

El sector agrícola en México ha sido objeto de múltiples y severas injusticias en materia de políticas públicas, sobre todo en aquello que concierne a los pequeños productores. Esto se refleja a lo largo de la historia del campo mexicano; una historia marcada por incongruencias y paradojas. Si bien en el discurso se ha reconocido la importancia del sector agrícola en su conjunto como un factor clave en el desarrollo económico nacional, en los hechos se muestra lo contrario, la política agrícola instrumentada por décadas sólo ha beneficiado a una pequeña parte de los productores.

En los primeros años de la década de los ochenta, el sector agropecuario otorgó muestras de competitividad y autosuficiencia, pero el abandono de políticas dirigidas al campo por la crisis estructural de la economía y por la incorporación al modelo neoliberal, asestaron un duro golpe al sector. La argumentación a favor de las nuevas medidas hacia el campo se centraba en el hecho de que la población económicamente activa del sector agrícola era relativamente muy grande respecto a su baja aportación al producto interno bruto (Ayala, et al., 2005: 130). Es decir, que el sector estaba operando de manera ineficiente, por lo que se requería extraer a la población improductiva y no competitiva del campo, suponiendo que simultáneamente se daría un proceso de industrialización que permitiría absorber a la población rural excedentaria, promesa que quedo alejada de la realidad.

Este nuevo escenario evidenció un sector agrícola vulnerable, cada vez más débil y empobrecido. Se experimentaba una crisis económica generalizada pero fue el sector agrícola el que resultó más afectado, particularmente, fueron los pequeños productores, descapitalizados quienes experimentaron los efectos más severos de la debacle económica.

La crisis económica tuvo como corolario un deterioro creciente en las condiciones de existencia de los productores agrícolas, experimentando una verdadera crisis de 
producción y reproducción social; enfrentándose a un acelerado proceso de pauperización (Martínez, 1991: 9). El incremento del desempleo, de la migración, la desnutrición, así como el aumento de empleos informales y precarios, son tan sólo algunos de los factores que contribuyeron al descenso permanente de su capacidad de producción y consumo de bienes y servicios de los productores agrícolas.

Con la idea de eliminar el lastre que impedía el "desarrollo rural" se emprendió una estrategia dirigida al desmantelamiento y desestructuración de la base productiva campesina. Entre las principales medidas se pueden mencionar las siguientes: 1) el abandono estatal y la desincorporación de los organismos de apoyo; 2) la contrarreforma agraria que permitió nuevas formas de acceso del capital al campo; 3) la introducción de empresas agroindustriales y el desplazamiento de los pequeños productores; y 4) la introducción de la biotecnología como un instrumento necesario dentro de la competitividad.

Las reglas del juego en materia agrícola han sido claras. Las políticas sectoriales ejemplifican lo que se está buscando: apoyar a los productores comerciales para reconvertir su economía hacia una más tecnológica, especializada y eficiente, en congruencia con la apertura comercial (Ayala, et al., 2005: 135). Mientras tanto, a los pequeños productores se les plantean dos posibles escenarios: integrarse al modelo económico actual y buscar competir bajo parámetros internacionales o buscar otra actividad para poder sobrevivir.

Algunos de los cambios más notables en el ámbito rural, consecuencia de la globalización neoliberal han sido las transformaciones en el mercado de trabajo. Actualmente, las familias rurales dependen cada vez más de ingresos producto de actividades no agrícolas, generando una ruptura en la visión tradicional que suponía que la sobrevivencia de las familias rurales dependía, en estricto sentido, de las actividades agrícolas y en donde las actividades no agrícolas eran complementarias.

En la actualidad, ya no se discute que la agricultura ha dejado de ser la actividad principal para la sobrevivencia económica en los hogares rurales. Durante décadas se insistió en que la actividad agrícola era el sector predominante, se constituía como el eje central que organizaba la vida social, económica y cultural en el medio rural. Hoy en día, es un hecho que las economías rurales dependen cada vez más de ingresos monetarios y actividades extraparcelarias muchas de ellas relacionadas con el ámbito urbano.

Otro de los aspectos que revelo la crisis agrícola fue el papel de la mujer en el medio rural. Consistente con lo anterior, Arias menciona que "las actividades que no eran agrícolas y que no eran practicadas por los hombres, pasaban, como se decía sin demostrarlo, a la categoría de complementarias" (Arias, 2009: 3). El trabajo de la mujer dentro y fuera de la parcela se había considerado como una ayuda, como algo complementario al trabajo del 
varón, a pesar de su importancia y función clave para la seguridad alimentaria de sus familias (Ballara, Damianović \& Valenzuela, 2012). Sin embargo, en este contexto y ante una lucha diaria por la sobrevivencia, se han ampliado y diversificados las fuentes de ingreso no agropecuarios así como la inclusión de un mayor número de mujeres a actividades remuneradas ya sea fuera o dentro del domicilio.

En este contexto la pluriactividad ${ }^{1}$ se consolida como una de las características actuales de los espacios rurales. Los aspectos que favorecen esta pluriactividad son diversos. Se pueden mencionar aquellos elementos que influyen en las transformaciones territoriales y económicas, entre las cuales destaca el cambio en la relación rural-urbana, como consecuencia de la reestructuración laboral y relocalización de actividades, teniendo claras consecuencias en la generación de nuevas alternativas de trabajo en el medio rural. La relación rural-urbana generó importantes cambios en el mercado de trabajo, estimulados principalmente por la cercanía a las ciudades, el incremento de vías de comunicación y medios de transporte.

También hay que considerar factores que aluden a las transformaciones en la dinámica social y económica de las familias rurales. Por ejemplo, la pérdida de rentabilidad de la actividad agropecuaria por parte de los pequeños productores, aunado a la persistencia y en algunos casos al incremento de los niveles de pobreza; la transición de una economía basada en el equilibrio entre lo que producían y consumían, a una economía en donde el ingreso monetario ha cobrado mayor importancia; las nuevas pautas de consumo y demandas asociadas a la educación de los hijos; las nuevas expectativas laborales y escolares principalmente entre los jóvenes (Arias, 2009: 296-297 y Gras, 2004: 102-103). Aspecto que se observan en mayor medida en las generaciones más jóvenes, quienes prefieren emplearse en actividades no agrícolas, pues el campo, además de ser poco rentable, les es ajeno (Méndez Sastoque, 2008). También cobra relevancia el cambio en las relaciones de género y, como se mencionó, el aumento de las mujeres en el mercado de trabajo.

Estos factores presentan un escenario complejo en el cual se gesta la pluriactividad, proceso que se concreta en la interacción entre el individuo y las decisiones dentro de la familia, con el contexto social y económico que los circundan. Cabe apuntar que la combinación entre las actividades agrícolas y no agrícolas, tienden a ser más intensas en la medida que son más complejas y diversas las relaciones entre los agricultores y el medio ambiente social y económico, en donde están ubicados.

${ }^{1}$ La pluriactividad para el ámbito rural se define como un proceso socioeconómico que implica la combinación de dos o más actividades laborales (una de las cuales es la agricultura) que desarrollan uno o más miembros de la familia rural. Estas actividades se pueden dar dentro o fuera del ámbito propiamente rural (Gras, 2004; Arias, 2009; De Grammont, 2008; Salas e Iñigo, 2014). 
En cuanto a la importancia de las redes de parentesco, Gras (2004) subraya que la pluriactividad es un atributo o cualidad de la familia ${ }^{2}$, aun cuando su observación se realice en el nivel de los individuos. En este sentido, el papel de la familia es de suma importancia, pues si bien la determinación de emplearse es una cuestión personal, es en el hogar en donde los distintos miembros discuten y tejen las posibles alternativas laborales, ya que el objetivo último es la sobrevivencia y reproducción. La pluriactividad se presenta como un proceso adaptativo socioeconómico que encierra la diversidad y complejidad en la esfera del trabajo y de las prácticas de sobrevivencia, como se mostrará en el caso de Tepetitla.

\section{Tepetitla de Lardizábal y la especialización productiva}

El municipio de Tepetitla de Lardizabal (ver figura 1) se ubica en la zona noroeste del estado de Tlaxcala y colinda al norte con el estado de Puebla y el municipio de Ixtacuixtla de Mariano Matamoros; al este con los municipios de Ixtacuixtla de Mariano Matamoros y Natívitas; al sur con el municipio de Natívitas y el estado de Puebla; y al oeste con el estado de Puebla. De acuerdo al censo del INEGI de 2010 cuenta con una población de 18,725 .

Figura 1.

Elaboración propia en base a INEGI (2010).

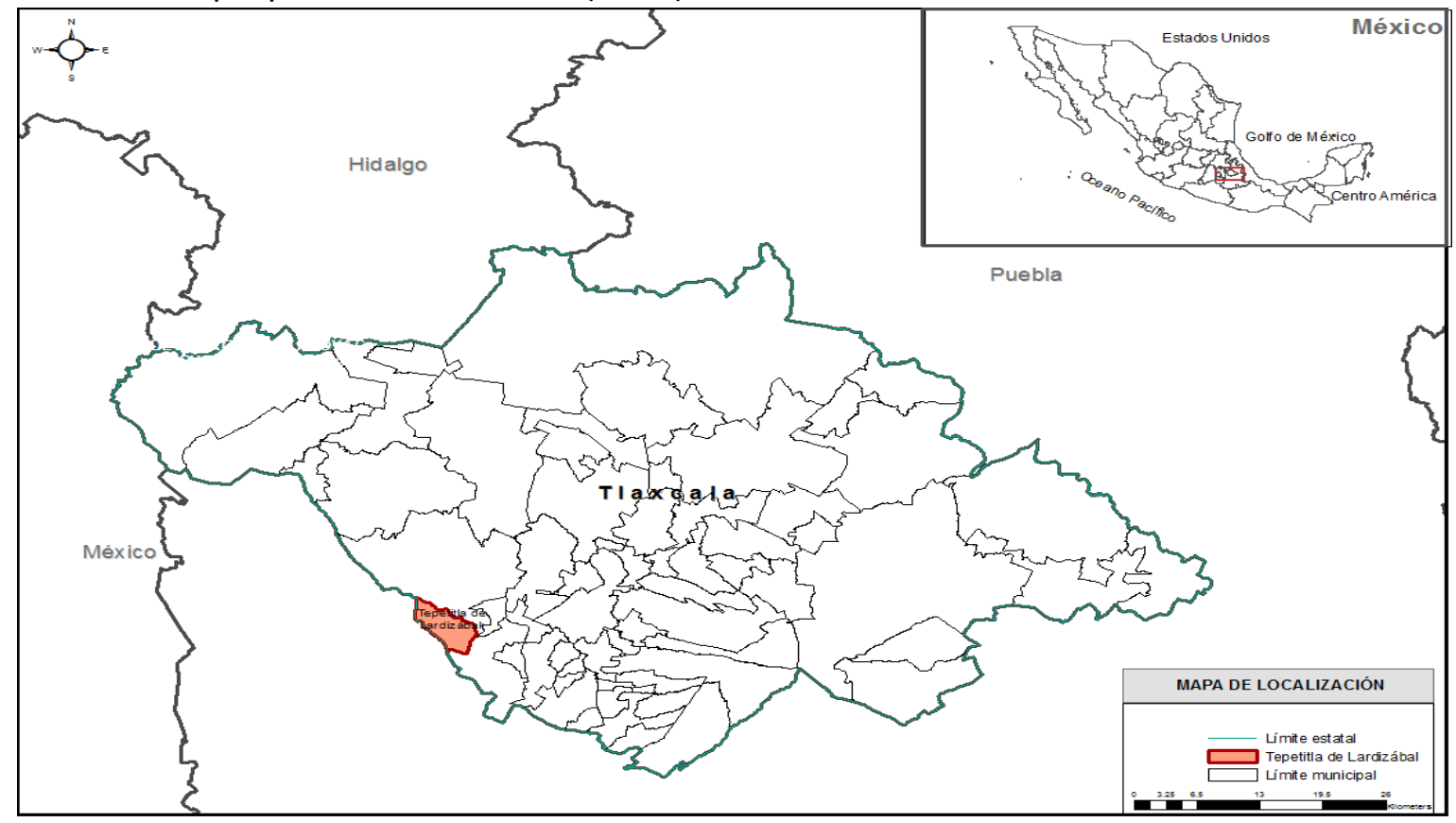

${ }^{2}$ La autora define a la "familia pluriactiva" cuando por lo menos uno de sus miembros está ocupado o realiza actividades diferentes a la propia producción agrícola, pudiendo o no dedicarse también al trabajo en la unidad económica (2004: 95). 
Aquí existe una importante presencia industrial afianzada en las últimas tres décadas, aunque es importante mencionar que en esta región una de las industrias más añejas ha sido la textil; hasta la década de los 60 sólo se tenía registro de estas viejas fábricas, posteriormente "entre los años de 1975 y 1980 que se instalan alrededor de 250 empresas (...). Se trataba de industrias nuevas y modernas que producían no sólo para el mercado nacional, sino que también exportan al mercado mundial. (...) Los parques, los corredores y las ciudades industriales (cambiaron) de manera definitiva la fase agraria y rural de la entidad." (Montiel, 2009:89).

Uno de los factores importantes que ha incentivado y afianzado el proceso industrial en la región ha sido la inversión en materia de infraestructura urbana, particularmente en caminos y carreteras. Cuenta de ello fue, en 1978, la inauguración del camino Santa Ana Chiautempan-Puebla, sobre esta ruta se establecieron industrias de aparatos electrodomésticos, loseta cerámica, piezas para la industria automotriz, alimentos balanceados para animales, calzado y fábricas textiles. También se inauguraron fábricas en la ruta san Martín Texmelucan-Tlaxcala, recientemente convertida en autopista, en la ciudad de Tlaxcala, y en los alrededores de Apizaco. De la misma forma la autopista México-Puebla, inaugurada a principios de los años sesenta, facilitó el traslado al Distrito Federal y reforzó aún más la hegemonía económica de la capital (Ibíd: 90).

El proceso de industrialización de esta región Tlaxcalteca no sólo se debe a factores internos, por el contrario, la urbanización e industrialización de los municipios vecinos del estado de Puebla han sido un factor clave para la transformación en ciernes de esta región. De acuerdo con Eisenblaetter, para 1970 se consolidaron nuevos polos industriales en el valle (Tlaxcala-Puebla) dejando atrás los municipios industrializados tradicionales como Atlixco (industria del algodón), Teziutlán (industria metalúrgica), Tehuacán (industria de bebidas y comienzos de industria de confección), Chietla (industria de transformación de azúcar), San Pedro Cholula (industria metalúrgica y textil), y la misma ciudad de Tlaxcala. El primer polo en destacar fue Cuauhtlancingo, que para esa década contribuía con un valor de la producción en Puebla del $12.9 \%$ y el municipio de San Miguel Xoxtla, que contribuye con el $9.5 \%$ del valor de producción estatal. Ambos colindan con el suroeste de Tlaxcala y su auge prácticamente se debe a la instalación de la Volkswagen en el primer municipio y la empresa siderúrgica HyLSA en el segundo. El tercero pertenece a Tlaxcala y es Papalotla de Xicohténcatl, destacado por su industria diversificada: textil, alimenticia y de bebidas, construcción, eléctrica, de motores y química (Velasco, 2014: 168).

Para el caso de la región de estudio, el proceso de industrialización se ha agudizado con la ubicación del corredor industrial Ixtacuixtla, el cual tiene presencia directa en los municipios de Tepetitla e Ixtacuixtla, aunque su influencia de atracción de mano de obra abarca de municipios aledaños. De acuerdo al directorio industrial del Estado de Tlaxcala, 
en el parque industrial Ixtacuixtla se ubican 13 empresas de las cuales seis pertenecen a la rama textil. Particularmente en el municipio de Tepetitla se tiene registro de tres empresas: una de la rama de alimentos, y dos de la rama textil, una de capital estadounidense dedicada a la fabricación de telas para tapicería automotriz y la otra de capital español y brasileño, dedicada a la fabricación de mezclilla (Gobierno de Tlaxcala, 2014). De esta manera se constata que el municipio de estudio ha estado sometido a intensos cambios producto del desarrollo urbano e industrial. Si bien existe una amplia diversificación industrial en la región, este trabajo se acota, como se señala en los objetivos, a la presencia de la maquila de confección. En Tepetitla se aprecia una importante especialización productiva dirigida a la confección de prendas de vestir, particularmente de pantalón de mezclilla.

La población ocupada total en el municipio es de 6,505. De acuerdo con datos del INEGI, de 1980 a 2010 se registró una caída de poco más del 60\% en la actividad primaria. Registrando para 2010 tan sólo el $\mathbf{1 7 . 2 0 \%}$ de la población ocupada en el sector primario, en tanto el sector secundario ocupó el $41.77 \%$, mientras que el sector terciario representó el $40.19 \%$ (12.18\% el comercio y $28.01 \%$ los servicios).

En el trabajo de campo se pudo observar la reducción de las actividades agrícolas, principalmente en las localidades en donde existe mayor presencia de talleres de confección y lavanderías como es el caso de la localidad de San Mateo Ayecac, perteneciente al municipio estudiado. En este caso en particular, además de la poca rentabilidad de los productos agrícolas se suma la presión urbana sobre el suelo agrícola. En el resto de las localidades aún hay presencia de parcelas dedicadas a la siembra, principalmente de maíz y hortalizas. El problema común en el municipio es el bajo precio de los productos agrícolas, y la dificultad para comercializar sus productos por los altos índices de contaminación del Río Atoyac, afluente con el cual muchos de los agricultores riegan sus cultivos.

Frente a la difícil situación del campo, que dicho sea de paso no es nueva ni exclusiva de la región, los pobladores crearon alternativas de sobrevivencia, la pluriactividad centrada en la migración y el empleo en el sectores industrial y de servicios, siendo la confección una de éstas actividades. De manera que, de acuerdo con los testimonios de los pobladores, la actividad económica relacionada con los talleres de confección no es un proceso reciente.

La confección de ropa, en su forma más tradicional, se ejerce desde los años ochenta, como lo señala la señora Gabriela:

Recuerdo que se empezó... ya tiene sus años, cuando venían de México con teloneros, los judíos, a encargar, en aquellos tiempos el pantalón de pana o playera, traían el corte y después venían o ya se los llevaban (...) allá por el centro por Mixcalco (...) esos fueron los primeros talleres (Gabriela, 2014). 
Si bien la actividad como tal tiene poco más de treinta años, ha sido en los últimos 15 años que se ha generado un auge de actividades relacionadas con la confección y procesos relacionados con las lavanderías y otras actividades como son los terminados, pegado de ojal, planchadurías, o actividades más específicas como elaboración de plantillas y aplicaciones para decorar los pantalones de mezclilla. Con el establecimiento de nuevas y diversas actividades, se generó una alta especialización productiva en el municipio.

De acuerdo al Directorio Estadístico Nacional de Unidades Económicas (DENUE, 2015) se tiene registrados 34 establecimientos relacionados con la manufactura de prendas de vestir, sin embargo, en trabajo de campo se pudo constatar que este número esta notablemente subestimado. El carácter informal de los talleres aunado a los conflictos ambientales, hace casi imposible tener un cálculo preciso del número de talleres, pues la gran mayoría de estos operan dentro de los hogares. Sin embargo, con los constantes recorridos de campo, se puede afirmar que el número de establecimientos sea más del triple de los contemplados en cifras oficiales.

El elevado número de talleres y lavanderías ha generado una importante demanda de trabajadores, en su mayoría jóvenes, quienes han encontrado en la costura una alternativa de trabajo. La manufactura de pantalones de mezclilla y en menor medida, la elaboración de trajes y vestidos se han distinguido por ser actividades redituables. Para muchas familias la costura se ha constituido como un verdadero "oficio" que se trasmite generacionalmente y les ha permitido construir un patrimonio.

El alto grado de especialización productiva en estas localidades y la alta demanda de mano de obra han generado una importante atracción de población de otras localidades e incluso de otros estados. Esta demanda de mano de obra ha hecho que se empleen tanto hombres como mujeres en la rama en general, no obstante, el mercado de trabajo al interior de la rama está claramente segmentado por género. Por ejemplo, los trabajos "más pesados" o que requieren mayor fuerza física son ofrecidos exclusivamente para hombres, al igual que las jornadas de trabajo con horarios nocturnos. Mientras que las tareas "más fáciles" o "delicadas" y aquellas que se pueden realizar en el domicilio y durante el día, son ocupadas por las mujeres.

Sin importar en que proceso de la cadena productiva o de comercialización se ubiquen las mujeres, es un hecho indiscutible la importancia de su participación en términos cuantitativos en la generación de ingresos de los hogares rurales.

Para las mujeres de la región el establecimiento de actividades industriales y de servicios ha significado una importante oferta laboral. Aunque esto no siempre resulta favorable: los empleos a los que en su mayoría tienen acceso son precarios y flexibles, con pocas o nulas prestaciones, jornadas exhaustivas y pocas oportunidades de desarrollo personal, 
además de que se multiplica el trabajo que realizan, ya que estos nuevos empleos se suman a su "obligada" jornada de trabajo en el hogar.

En este tenor, Guadarrama ofrece un análisis interesante de la incorporación de las mujeres al mercado de trabajo:

(...) la incorporación de las mujeres al mercado de trabajo creció considerablemente en este contexto de apertura y flexibilización de las relaciones laborales. (...) La discusión sobre las formas de inserción femenina en el mercado de trabajo, implica reconocer los estereotipos sociales incubados desde la familia y la escuela y reproducidos en el mercado de trabajo, es también parte de una discusión sobre las formas de trabajo flexibles feminizadas que profundizan las desigualdades en la división sexual del trabajo doméstico y extradoméstico. Una prueba de ello es que el trabajo femenino se ha concentrado en los servicios de menor paga, lo cual permite también que las mujeres continúen tomando la mayor responsabilidad del trabajo doméstico. En otras palabras, el trabajo flexible en su forma más pura, como empleo fluido y temporal, excluyente, vulnerable, con riesgos, es el trabajo ejecutado por las mujeres (Guadarrama 2008: 336).

Con lo anterior se puede decir, que el mercado de trabajo está conformado tanto por variables económicas producto de la globalización neoliberal, políticas propuestas y ejecutadas por los distintos niveles de gobierno, y también está permeado por las normas sociales y culturales propias de las regiones y poblaciones.

De tal manera que se pueden identificar aspectos socioculturales que no favorecen el entorno laboral de las mujeres; por una parte, los estereotipos sociales son trasladados al ámbito laboral, en los trabajos se les adjudican las labores menos cualificadas, más "sencillas y delicadas" con las implicaciones salariales que conlleva, además de hacer diferencias basadas en su condición civil y materna (discriminación negativa). Se observó que las mujeres jóvenes solteras y sin hijos pueden acceder a mejores puestos laborales, caso contrario a las mujeres casadas y con hijos. Es decir, las oportunidades laborales no las determinan sus capacidades sino que son limitadas de acuerdo a los códigos sociales y culturales asignados por cuestiones de género y condición civil.

\section{El trabajo de las mujeres en los talleres de confección}

Comenzaré citando a Girón (2009), quien retomando a Beneria, señala la importancia del papel de las mujeres en el proceso del desarrollo económico y el abatimiento de la pobreza a partir de su incorporación al mercado global en el marco de las políticas neoliberales. Apunta que este proceso ha ido acompañado de una serie de contradicciones y paradojas en el desenvolvimiento del desarrollo no sólo de las economías emergentes sino específicamente de ciertos sectores en los que las mujeres se 
emplean y en los cuales, en muchas ocasiones ganan pero también pierden. Dentro del ganar se puede considerar el mejoramiento de la autoestima, la mayor libertad personal y la incorporación a situaciones de autonomía económica y a nuevos circuitos de relaciones sociales, que afectan de forma directa las dinámicas familiares (Fawaz y Soto, 2012).

Con la incorporación de las mujeres al mercado de trabajo se logra cierta independencia económica, lo cual implica, en buena parte de los casos, una mejor condición social para las mujeres. La autonomía financiera que proporciona el trabajo asalariado, fortalece la posición de las mujeres dentro de la estructura familiar; contribuye al bienestar de sus familias y es un aliciente fundamental (Chávez, 2010: 84). No obstante, las condiciones en el mercado de trabajo aún están marcadas, con sesgos de género, que no benefician a las mujeres, por el contrario, hoy por hoy el grueso de las mujeres participan en este mercado en desventaja frente a los hombres.

En el caso de estudio es interesante señalar que de acuerdo al cuestionario aplicado el $51 \%$ de los trabajadores en los talleres de confección son varones frente al $49 \%$ de mujeres. Mientras que en las lavanderías, un espacio de trabajo masculinizado, es del $72 \%$ de hombres frente al $28 \%$ de mujeres. Este dato resulta interesante porque el trabajo en las maquiladoras de confección se ha caracterizado por ser un espacio feminizado, sin embargo, la falta de empleo ha generado mayor competencia en el mercado, de tal manera que los empleos son ocupados en buena parte por los varones, quienes tienen más oportunidades de ser contratados que algunos sectores de mujeres. Así lo señala un dueño de taller:

En tiempo de alta hay mucho trabajo, las mujeres son responsables pero si son casadas o con hijos tienen más faltas, en cambio un hombre es más libre de su tiempo, puede disponer porque tiene mujer en casa que atienda a los hijos. Por eso prefiero contratar hombres (Gregorio, 2015)

Además de enfrentar desventajas por género al momento de la contratación se registró que en los talleres las mujeres ocupan los trabajos menos calificados, los más precarios y peores pagados, además de que existe mayor probabilidad de prescindir del trabajo de las mujeres o de justificar su despido, es decir, están más expuestas al desempleo, por ejemplo, son comunes los despido bajo el argumento de que "falta mucho por sus hijos" o "ya no la dejó el marido".

A pesar de que las mujeres han logrado incidir en el mercado de trabajo, incluso en nichos laborales considerados para hombres, como es el caso de las lavanderías, el papel de los cuidados del hogar, hijos, ancianos y enfermos es una tarea casi exclusiva de ellas. Este aspecto genera un aumento de la carga de trabajo, sobre todo para las mujeres que laboran fuera del hogar o que realizan trabajos remunerados por su cuenta en su domicilio, ejercen dos o más jornadas de trabajo. 
El trabajo de reproducción y del hogar está socialmente asignado a las mujeres y nunca es abandonado. Esta condición fundamental define su acceso al mercado laboral en circunstancias desfavorables respecto a los hombres, en cuanto a la calidad del empleo, posibilidad de ascenso, y desde luego, salarios. Las características de la oferta de trabajo femenina necesariamente deben incluir aspectos relacionados con el trabajo doméstico y la organización familiar, es decir, características que traspasan las fronteras del hogar para introducirse en el mercado laboral. Como señala Chávez (2010) del seno familiar parte la segregación contra las mujeres, que además, se traduce en un exceso de trabajo global, que rebasa por mucho el trabajo global de los hombres, dentro y fuera del hogar.

La búsqueda por la obtención de ingresos es similar entre las mujeres solteras o casadas, con hijos o sin hijos, aumentado esta necesidad en las jefas de familia solteras con hijos. El desempleo no es opción entre quienes son proveedoras, de manera que es más probable que se auto-empleen o acepten empleos que les generen ingresos marginales, o accedan a trabajos precarios pero que les garanticen algún nivel de ingresos. Si bien es cierto que la decisión por emplearse está mediado por la necesidad de percibir ingresos monetarios, también se observa que ésta opción no es una decisión individual y unilateral, sobre todo en las mujeres casadas y las solteras que aún viven en casa de sus padres o familiares.

La opción de ejercer algún tipo de trabajo remunerado fuera del hogar atraviesa por una serie de negociaciones. Como señala una entrevistada:

No nos alcanzaba el dinero porque ya con dos hijos es difícil (...) antes cuando era más chica trabajé en la maquina con mi mamá, y entonces le dije (a su esposo) yo me voy a trabajar porque con lo tuyo no alcanza y parece que le eche una cubeta de agua fría. Se ofendió, casi me pega, pero yo insistí hasta que lo convencí (...) tengo mi dinero, bueno, es dinero para todos le digo, pero sí costó mucho que ya de casada me pusiera a trabajar (2014).

Calveiro (2005) insiste en que analizar las relaciones de poder entre hombres y mujeres, como identidades de género construidas socialmente, implica observar precisamente cómo se articulan e interactúan uno frente al otro, el hombre frente a la mujer o en relación con ella, y viceversa. Particularmente en la familia no hay una y ultima confrontación, según la cual se alinean los actores, sino redes de relaciones de poder en las que un mismo sujeto juega de maneras diversas. Por ejemplo, - en el marco de las relaciones sociales de género desventajosas- la mujer puede ocupar, a la vez, una posición subordinada en relación con su pareja y una posición de poder en relación con sus hijos, pero también con las nueras o con otras mujeres del mismo núcleo. Estas distintas posiciones en las relaciones de poder no se pueden remitir a una confrontación última sea ésta la que existe entre hombres y mujeres, entre padres e hijos o cualquiera otraque pudiera funcionar como clave explicativa final. Así, el mismo actor puede funcionar 
como sujeto de poder y como sujeto resistente según la relación a que se refiera.

Los estudios de género han trabajado de manera abundante la relación de género y poder, una buena parte enfatiza una perspectiva "lineal" y descendente del poder ejercido de los hombres hacia las mujeres que, hasta cierto punto simplifica el problema. En realidad en los hogares, esto es más complejo, por ejemplo, en el caso en donde las mujeres viven con las suegras, son éstas las que ejercen coerción y en muchos casos violencia hacia las nueras cuando éstas quieren salir a trabajar. También es el caso de las hijas a quienes se les imponen mayores cargas de trabajo doméstico que a sus pares varones y se presenta un mayor control sobre sus tiempos fuera del hogar. Estas acciones en su mayoría son llevadas a cabo por las madres quienes poseen un poder legitimado sobre los hijos y en general en el núcleo familiar.

Así lo señalan dos testimonios, el primero de Laura quien comenta el caso de su nuera:

Mi hijo se accidentó y en un principio aquí no les faltaba un taco, pero ella no estaba convencida, que le hacía falta el dinero (...) yo le decía que no, que qué buscaba en la calle, pero no se convenció, y mírela anda en el taller trabajando. ¿Y qué me queda? ver a mis nietos, eso si aquí se llega a lo hora o no se entra. Ella tiene que obedecer mis reglas, porque está bajo mi techo (2015).

Sandra, estudiante y trabajadora:

Trabajo medio tiempo entre semana porque voy a la escuela sábados y domingos todo el día (...) muy pesado porque tengo que ayudar a mi mamá a echar tortilla y llevarle de comer a los puercos, luego la escuela o lo que le haga falta a mi mamá (...) mi mamá no quería que trabajara porque me dijo -te va a gustar el dinero y ya no vas a querer la escuela- pero yo le digo -ay mamá a poco crees que me gusta estar sentada todo el día- (...) me dejó con la condición de que no dejara la escuela y le pasara ayudar al quehacer de la casa (2015).

Como señala Calveiro, los hogares no son un conjunto indiferenciado de individuos que comparten condiciones de igualdad en actividades relativas al mantenimiento y reproducción de la unidad. Se trata más bien de un microcosmos anclado en pautas organizativas que se basan en relaciones de poder. Generan no sólo consensos y acciones solidarias, sino también conflicto y lucha (2010:33) tensiones, resistencias y confrontaciones, a veces casi imperceptibles que se viven en lo cotidiano.

En el caso de este municipio rural, a las mujeres se les continúa asignado trabajos de reproducción y cuidado. Cuando se requiere su incorporación al trabajo asalariado existe un consenso generalizado de que es mejor que trabaje en el domicilio o dentro de la propia localidad. La movilidad laboral femenina fuera del municipio es poco ejercida. 
Como menciona una entrevistada "me gusta ganar mi dinero pero no me gusta estar peleando con mi esposo, por eso mejor que me traigan el trabajo a casa". El trabajo a domicilio es una actividad común en Tepetitla, sin embargo, esto ha implicado una reorganización en el tiempo de las mujeres, lo cual implica una creciente autoexplotación que pone al límite la salud y la calidad de vida de ellas. Trabajar dentro de casa requiere de la combinación de actividades y espacios simultánea. Se trabaja cuando los hijos están en la escuela o por las noches cuando éstos duermen. Se tiene registro de mujeres que en temporada de mayor demanda, que es entre los meses de noviembre y febrero, duermen en promedio dos horas. Como el caso de Julia, quien trabaja por destajo en casa:

En temporada alta tienes que aprovechar porque hay veces que no te dan trabajo porque no hay. Yo aprovecho y hasta de dos clientes recibo trabajo (...) se duerme poco en esas fechas porque hay que entregar el trabajo, yo llego a entregar hasta cien piezas y me las tengo que hacer en los ratos libres (...) yo prefiero coser de un jalón en las noches que nadie me moleste, ni los hijos, ni el marido, ni la vecina, me dedico a darle a la máquina toda la noche.

Es importante hacer mención que el trabajo en los talleres es ocupado principalmente por jóvenes y adultos-jóvenes. De acuerdo con los datos recabados, el rango de edad que concentra mayor porcentaje de personas trabajando en talleres es de 15 a 45 años sin distinción de sexo. Conforme avanza la edad es más probable que las mujeres queden fuera del trabajo en el taller. Las mujeres adultas y ancianas son las encargadas del trabajo agrícola que aunque poco aún se ejerce en el municipio. Como se mencionó, el tipo de agricultura que permanece en el municipio es principalmente para el autoconsumo, destacando el cultivo de maíz y de algunas hortalizas. El maíz por lo regular es consumido por la familia para la elaboración de tortillas y para alimento de animales de traspatio, mientras que las hortalizas una parte es para el autoconsumo y otra es comercializada ya sea en el mismo municipio o en zonas aledañas. Si bien es cierto que para la mayoría de los pobladores los ingresos provienen de las diversas actividades en los talleres y lavanderías, el trabajo agrícola continúa siendo una actividad productiva que amortigua los gastos cuando el trabajo en los talleres disminuye. A pesar de su importancia coyuntural no existe un reconocimiento social generalizado de esta ocupación, el trabajo ejercido por las mujeres en el campo se continúa considerando una "ayuda" para la familia; además de que la propiedad de la tierra sigue siendo en su gran mayoría de los hombres.

\section{Reflexiones finales}

Las transformaciones rurales en el ámbito social y económico han favorecido la inserción de las mujeres en el mercado de trabajo, cada vez más diverso. Las condiciones más adversas del campo mexicano han orillado a la integración de más miembros de las familias en la búsqueda de ingresos que les permita garantizar su sobrevivencia. En esta 
búsqueda se encuentran con un problema fundamental: la ausencia de trabajos de buena calidad y condiciones laborales aceptables.

La necesidad de tener fuentes de ingresos, junto con la inexistencia de un seguro de desempleo, obliga al conjunto de trabajadores y en especial a las mujeres a aceptar trabajos en condiciones precarias o generar sus propios trabajos. Las evidencias han mostrado consistentemente que ante un panorama desempleo generalizado, que afecta tanto a mujeres como a los hombres, persiste una mayor vulnerabilidad entre las mujeres, no sólo a caer en la desocupación sino a tener ocupaciones de menor calidad que los hombres.

A pesar de este escenario las mujeres han ganado terreno en el mercado laboral, participando en mayor grado como proveedoras de ingreso familiar con su inserción en la economía formal o en la informal, sin que esto signifique un desprendimiento del trabajo doméstico, aumentando con ello su carga de trabajo y generando mayores condiciones de inequidad frente a los hombres.

La incorporación al mercado de trabajo no ha ido acompañada de un cambio cultural, o este ha cambiado de manera gradual. En la segmentación laboral por género persisten las connotaciones negativas hacia el trabajo ejercido por las mujeres. De tal manera que se pueden identificar aspectos socioculturales que no favorecen el entorno laboral de las mujeres; por una parte, los estereotipos sociales son trasladados al ámbito laboral, por lo que en la gran mayoría de trabajos se les adjudican las labores menos cualificadas, más "sencillas y delicadas", y por ser actividades sencillas son menos remuneradas. Por otra parte, las diferencias basadas en su condición civil y materna han generado una discriminación negativa. Destacando el grupo de las mujeres casadas y con hijos como las más afectadas y con mayores tensiones y conflictos a nivel familiar, sobre todo con la pareja. A pesar de que parece existir un mayor consenso entre las parejas de que el cuidado de los hijos corresponde a ambos, en el momento en que las mujeres deciden trabajar fuera de hogar se genera el conflicto, además del argumento del cuidado de los hijos, subyace la idea de que "el lugar" de la mujer está en el ámbito privado, dentro del hogar, así el trabajo en casa es aceptado y genera menores tensiones familiares. Un testimonio de un varón es muy claro en este sentido: "Le digo a mi mujer que yo no me opongo a que trabaje, que es bueno que ella tenga su dinero, pero que le traigan el trabajo aquí a la casa, si aquí tiene la máquina, que busca fuera" (A.A. 2016).

Algunas mujeres casadas y empleadas en talleres, señalaron que percibían mayor participación de sus parejas en el cuidado de sus hijos cuando ellas estaban dedicadas únicamente a su hogar. Además de los conflictos o tensiones generadas en el hogar, este grupo de mujeres tienen mayor problema para ajustar sus horarios de trabajo siendo vulnerables a los despidos y quedando más expuestas al desempleo. Por otra parte, se constató que las mujeres jóvenes solteras y sin hijos pueden acceder a mejores puestos 
laborales compitiendo casi en igualdad de condiciones frente a los hombres aunque como se mencionó al ser solteras tienen que colaborar en mayor medida en las tareas del hogar para "compensar su ausencia".

Lo anterior permite la reflexión sobre los cambios ocurridos en el entorno familiar como consecuencia de procesos que traspasan la escala local. Así podemos observar como el proceso de globalización y modernización, los cambios en las políticas públicas generan trasformaciones productivas y sociales que inciden en las dinámicas laborales tal es el caso de la pluriactividad que si bien ha estado presente en las sociedad rurales, en la actualidad se observa una mayor diversificación de ocupaciones así como un incremento en la participación de otros miembros de la familia (mujeres, ancianos y niños) al mercado de trabajo ya sea para garantizar la sobrevivencia o por la existencia de proyectos personales y dinámicas económicas familiares.

El avance hacia relaciones más igualitarias implica el visibilizar la importancia del trabajo de la mujer dentro y fuera del hogar. Considero que un primer paso es reconocer que las actividades "no remuneradas" como el trabajo de reproducción, las labores domésticas y los cuidados del hogar, requieren de una cantidad de tiempo considerable y son fundamentales para la producción y reproducción de la mano de obra dentro de los espacios rurales y en general en la sociedad. Un segundo punto es visibilizar la importancia del ingreso salarial proveniente de los trabajos extradomésticos para la sobrevivencia de las familias rurales. Se trata de poner a la discusión la necesidad enfrentar los cambios que se requieren para, además de reconocer el trabajo de las mujeres, tratar de disminuir los conflictos y desigualdades en los distintos ámbitos sociales. Finalmente, también se debe poner en debate el sentido del trabajo para las propias mujeres, en el caso de estudio poco se vincula con los logros y satisfacciones personales, por el contrario existe una fuerte connotación de beneficio familiar. 


\section{BIBLIOGRAFÍA}

Arias, Patricia (2009). Nueva estructura Ocupacional en el campo Latinoamericano, en Grammont y Martínez (coords.): La pluriactividad en el campo latinoamericano, $p$. 171-206. Quito: FLACSO.

Ayala Ortiz, Dante Ariel y Solari Vicente Andrés (2005). México y Estados Unidos, análisis comparativo de dos crisis agrícolas, en Espiral, año/vol. XII, septiembre-diciembre, No. 034 , p. $125-146$.

Ballara, Marcela; Damianović Ninoska y Valenzuela Rodrigo (2012). Mujer, agricultura y seguridad alimentaria: una mirada para el fortalecimiento de las políticas públicas en América Latina, BRIDGE development - gender. Disponible en: http://www.fao.org/family-farming/detail/es/c/317278/

Beneria, Lourdes (2006). La aparición de la economía feminista, en Historia agraria, No. 17, p. 59-61.

Calveiro, Pilar (2005). Familia y poder. Buenos Aires: Libros de la Araucaria.

Chávez Hoyos, Marina (2010). Trabajo femenino, las nuevas desigualdades. México: Instituto de Investigaciones Económicas/UNAM.

De Grammont, Hubert Carton de (2008). El concepto de nueva ruralidad, en Pérez, Farah y Grammont (Coords.) La nueva ruralidad en América Latina, p. 23-44. Bogotá: FLACSO, Pontificia Universidad Javeriana.

Directorio Estadístico Nacional de Unidades Económicas (2015). Disponible en: http://www3.inegi.org.mx/sistemas/mapa/denue/default.aspx

Echánove Huacuja, Flavia y Steffen Riedemann Cristina (2005). Globalización y reestructuración en el agro mexicano: los pequeños productores de cultivos no tradicionales. México: Universidad Autónoma de Chapingo, Plaza y Valdés.

Fawaz, Julia y Soto Paula (2012). Mujer, trabajo y familia. Tensiones, rupturas u continuidades en sectores rurales de Chile central, en La Ventana, número 35, p. 218-254.

García, Brígida (2001). Reestructuración económica y feminización del mercado de trabajo en México, en Papeles de la población, enero-marzo, No. 27, p. 45-61.

Girón, Alicia (coord.) (2009). Género y globalización. Argentina: CLACSO. 
Gobierno de Tlaxcala (2014). Directorio Industrial. Disponible en: http://www.contactopyme.gob.mx/parques/empresa.asp?ID=118

Gras, Carla (2004). Pluriactividad en el campo argentino: el caso de los productores del sur santafecino, en Cuadernos de Desarrollo Rural, No. 51, p. 91-114.

Guadarrama Olivera, Rocío (2008). Los significados del trabajo femenino en el mundo global. Propuesta para un debate desde el campo de la cultura y las identidades laborales", en Estudios Sociológicos, Vol. XXVI, No. 77, p. 321-343.

Hernández, Roberto; Fernández, Carlos y Baptista María del Pilar (2003). Metodología de la Investigación. México: Mc. Graw Hill.

INEGI (2010). Censo de Población y Vivienda. Principales resultados por localidad. Disponible en: http://www.inegi.org.mx/sistemas/consulta resultados/iter2010.aspx?c=27329\&s= est

INEGI (2013). Mujeres y hombres en México 2013, Aguascalientes: INEGI.

Martínez Borrego, Alma Estela (1991). Organización de productores y movimiento campesino. México: Siglo XXI.

Méndez Sastosque, Marlon (2008). La construcción de mixturas rural-urbanas: una lectura subjetivizante, en Cuadernos de Desarrollo Rural, Vol.1, No. 54, p.129-152.

Montiel Torres, Oscar (2009). Trata de personas: padrotes, iniciación y modusoperandi. México: INMUJERES.

Morales, Josefina (2000). Maquila 2000, en Morales (coord.) El eslabón industrial. Cuatro imágenes de la maquila en México, p. 17-102. México: Nuestro Tiempo.

Salas, Hernán y González Iñigo (2014). La reproducción de la pluriactividad laboral entre los jóvenes rurales en Tlaxcala, en Papeles de Población, volumen 20, p. 281-307.

Velasco Santos, Paola (2014). Antropología socioambiental. Ecología política, sujetos rurales y transformación del río Atoyac en el municipio de Nativitas, Tlaxcala. Tesis de Doctorado en Antropología. México: Universidad Nacional Autónoma de México, Instituto de Investigaciones Antropológicas. 